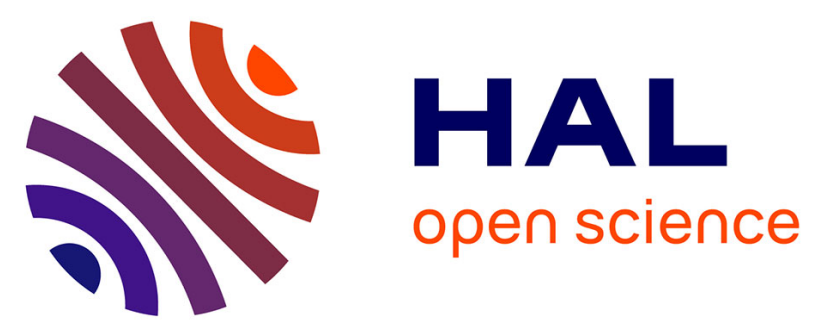

\title{
State-of-strain evaluation with fiber Bragg grating rosettes: application to discrimination between strain and temperature effects in fiber sensors
}

Sylvain Magne, Stéphane Rougeault, Manuel Vilela, Pierre Ferdinand

\section{- To cite this version:}

Sylvain Magne, Stéphane Rougeault, Manuel Vilela, Pierre Ferdinand. State-of-strain evaluation with fiber Bragg grating rosettes: application to discrimination between strain and temperature effects in fiber sensors. OSA Applied Optics - Information Processing, 1997, 36, pp.9437-9447. cea-01840677

\section{HAL Id: cea-01840677 https://hal-cea.archives-ouvertes.fr/cea-01840677}

Submitted on 16 Jul 2018

HAL is a multi-disciplinary open access archive for the deposit and dissemination of scientific research documents, whether they are published or not. The documents may come from teaching and research institutions in France or abroad, or from public or private research centers.
L'archive ouverte pluridisciplinaire HAL, est destinée au dépôt et à la diffusion de documents scientifiques de niveau recherche, publiés ou non, émanant des établissements d'enseignement et de recherche français ou étrangers, des laboratoires publics ou privés. 


\title{
State-of-strain evaluation with fiber Bragg grating rosettes: application to discrimination between strain and temperature effects in fiber sensors
}

\author{
Sylvain Magne, Stéphane Rougeault, Manuel Vilela, and Pierre Ferdinand
}

\begin{abstract}
An optical rosette that incorporates fiber Bragg gratings as strain gauges has been designed, fabricated, and tested. We investigated it by measuring the state of strain of a thin plate as the test structure submitted to an increasing load in a four-point bending configuration and for various angular orientations. This device has also been successfully investigated as a self-temperature-compensated in situ uniaxial strain sensor without any angular dependence and with high accuracy in recovery analysis, leading us to expect many industrial applications. Printed circuit processes or integrated optics on polymers would provide a means for accuracy, reproducibility, and integration in a mass-produced process. (C) 1997 Optical Society of America

Key words: Fiber Bragg grating, rosette, optical fiber sensor, smart material and structure, strain and stress analysis, temperature compensation.
\end{abstract}

\section{Introduction}

Fiber Bragg gratings (FBG's) are now recognized as important optoelectronic components for guidedwave optics, owing to the large number of device functions in which they can be used.1,2 They are used extensively in telecommunications (wavelengthdivision multiplexing, gain flattening of erbiumdoped fiber amplifiers, and dispersion compensation), in instrumentation (wavelength-selective reflectors for fiber lasers and, to a lesser extent, semiconductor lasers), and in sensors for the measurement of strain, temperature, and hydrostatic pressure. ${ }^{3}$

The first reported Bragg grating (the so-called Hill grating) dates back to 1978 when Hill and co-workers launched light at $488 \mathrm{~nm}$ from an argon-ion laser into a germanosilicate optical fiber. ${ }^{4}$ In those experiments the grating was formed by the standing-wave interference pattern set up by counterpropagating beams inducing periodic perturbations of the refractive index along its length, and the center Bragg

\footnotetext{
The authors are with the Départment d'Electronique et d'Instrumentation Nucléaire, Service de Physique Electronique, Laboratoire d'Electronique de Technologie et d'Instrumentation, Commissariat à l'Energie Atomique (Technologies Avancées), Centre d'Etudes de Saclay, 91191 Gif-sur-Yvette Cedex, France.

Received 1 April 1997; revised manuscript received 16 July 1997. 0003-6935/97/369437-11\$10.00/0

(C) 1997 Optical Society of America
}

wavelength equaled the laser wavelength. Because of the long interaction length, narrow-band Bragg reflection filters were formed with reflectivities approaching $100 \%$. After that, in spite of the significant interest raised at the time, few studies were pursued until 1989 when Meltz et al..$^{5}$ reported that gratings could be written by two-beam holographic exposure through the side of the fiber by use of UV radiation. This method dramatically improved the writing efficiency because of the direct photoionization process induced by UV light (in contrast with the Hill experiment, in which photoionization resulted from a two-photon absorption process). Moreover, this method allowed the possibility of producing gratings with an arbitrarily selected Bragg wavelength simply by adjusting the angle between the exposed beams with respect to the fiber axis. Although the free-space holographic method (Meltz's method) allowed great flexibility in fabrication of FBG's, a higher reproducibility was achieved by a method based on near-contact exposure through a phase mask. ${ }^{6}$ In this case the grating was photowritten by interference of -1 and +1 diffraction orders with a period equal to half of that of the mask independent of the exposure wavelength. Since then activity in photo-induced Bragg gratings has increased rapidly, leading to the demonstration of many types of grating (moiré, chirped, phase-shifted, etc.) ${ }^{1,2}$ in fibers or on substrates and in many materials, such as germanosilicate glasses with or without hydrogen loading; ${ }^{7}$ 
cerium-, terbium-, or tin-doped silicate glasses; $; 89$ cerium-doped fluorozirconate glasses; 10 and polymers. ${ }^{11}$

FBG's are known to be well suited for measuring strain and temperature, e.g., in smart structures, ${ }^{12-14}$ and have unique advantages over classical electrical strain gauges (metal-foil resistance and piezoelectric ceramic strain gauges). These advantages are at first conveyed by intrinsic features of optical fiber sensors, such as electromagnetic interference immunity, light weight and small size, high temperature and radiation tolerance, flexibility, stability, and durability against harsh environments. FBG's have the advantages of being absolute, linear in response, interrupt immune, and of low insertion loss so that they can be multiplexed in a series on a single-mode fiber. The spectral signature renders the measurement free of intensity fluctuations. This is a great asset for long-term reproducibility. Moreover, FBG's can be easily embedded into composite materials and can provide local damage detection as well as internal strain field mapping with high resolution in strain and localization and a large measurement range. The FBG is therefore a major component in the development of fiber-optic smartstructure technology. It offers the promise of undertaking real-time structural measurements with built-in sensor systems expected to be cost effective when the number of sensors that can be multiplexed is large.

In the scope of making strain measurements using Bragg gratings, one can design FBG rosettes following the example of electric strain rosettes. Rosettes are made of two or three noncollinear strain gauges mounted on a common substrate, typically arranged at $45^{\circ}$ or $120^{\circ}$ with respect to one another to form rectangular or delta rosettes, respectively. They are used extensively in experimental stress analysis to measure the two principal strains (and stresses) and the orientation of the principal axis whenever it is not known a priori. Such a concept has already been demonstrated with fiber interferometer strain gauges $^{15-17}$ and fiber Fabry-Perot strain gauges placed onto common substrates ${ }^{18}$ or embedded in neat resin and graphite-epoxy composite laminates for mapping of two-dimensional strain fields. ${ }^{19}$ To our knowledge rosettes have not yet been demonstrated with FBG's as strain gauges. FBG rosettes have several advantages over interferometric strain rosettes, including the fact that they are potentially easy to manufacture (amenable to batch-process manufacturing) and the multiplexing capability of Bragg grating-based rosettes and gauges in a series along a single monomode fiber.

However, whatever the method used, temperature sensitivity of the fiber sensor can complicate its application as a strain gauge. Let us focus on Bragg grating-based optical sensors: many solutions have been proposed to eliminate their thermal-apparent strain. The most obvious solution lies in compensating for the temperature influence by using another FBG (reference grating) shielded from strain (e.g., by
Teflon sleeving) that measures only temperature. The Bragg wavelength of this grating is then subtracted from that of the FBG-measuring strain and temperature. ${ }^{20}$ This correction is fully efficient if both transducers are at the same temperature (i.e., they are close enough) or if the difference in temperature remains constant.

We now attempt to make a survey of in situ temperature-compensating methods for Bragg gratings. They can be classified according to whether they are intrinsic (i.e., make use of the fiber properties themselves) or extrinsic (i.e., require an extra material). At least eight intrinsic methods have been proposed. The first method involves the use of a saturated chirped grating ${ }^{21}$ of which the spectral bandwidth increases with strain, thus increasing the return signal. The second method involves the use of two superimposed Bragg gratings of different center wavelengths. ${ }^{22,23}$ A third method relies on the use of a Bragg grating photowritten in a highly birefringent fiber. ${ }^{24}$ A fourth method involves the use of two Bragg gratings of close center wavelengths photowritten on either side of a splice between two fibers of different cladding diameters (80 and $120 \mu \mathrm{m}){ }^{25} \mathrm{~A}$ fifth method relies on the primary and second orders of diffraction from a single Bragg grating. ${ }^{26}$ A sixth method makes use of the dependence of rocking filters on strain and temperature. ${ }^{27,28} \mathrm{~A}$ seventh method involves the use of two Bragg gratings and a long-period grating, ${ }^{29}$ and an eighth method relies on the use of a FBG and a Fabry-Perot interferometer. ${ }^{30}$ The first method relies on an intensity measurement (4- $\mu \varepsilon$ resolution in strain), and temperature is not measured. For any of the seven latter methods temperature and strain information can be recovered, provided that two linear equations, both functions of temperature and strain, can be solved. ${ }^{31}$

Conversely, at least two extrinsic methods have been proposed. The first makes use of a cantilever with two FBG's surface mounted on opposite surfaces (top and bottom). ${ }^{32}$ One grating is stretched while the other is compressed. The difference in Bragg grating center wavelengths is temperature independent because both Bragg gratings have the same thermal sensitivity. The second method consists of a passive temperature-compensating package and makes use of a proper choice of materials and dimensions to nullify the temperature-to-wavelength coefficient. ${ }^{33,34}$ These two methods involve a mechanical compensation and do not provide a temperature measurement.

Here we describe the design, fabrication, and test of a Bragg grating rosette. This device was tested in two ways. The first test was dedicated to the measurement of the state of strain of a plate as the test structure. The second test was more unexpected, as it provided a rigorous measurement of a uniaxial strain (or stress) independent of both temperature and orientation of the rosette onto the test structure. 


\section{Bragg Grating Properties}

The Bragg center wavelength of a grating $\lambda_{g}$ is given by the Bragg phase-matching condition

$$
\lambda_{g}=2 n_{\mathrm{eff}} \Lambda,
$$

where $\Lambda$ is the fringe spacing of the grating and $n_{\text {eff }}$ is the effective refractive index of the $L P_{01}$ mode (depending on the $V$ value of the fiber). The relative change in the center Bragg wavelength is therefore

$$
\frac{\Delta \lambda_{g}}{\lambda_{g}}=\frac{\Delta n_{\text {eff }}}{n_{\text {eff }}}+\frac{\Delta \Lambda}{\Lambda} .
$$

Both refractive index and spacing depend on temperature and strain, as follows:

$$
\frac{\Delta \lambda_{g}}{\lambda_{g}}=\varepsilon_{\text {ax }}-\frac{n_{\text {core }}^{2}}{2}\left[\varepsilon_{r}\left(P_{11}+P_{12}\right)+P_{12} \varepsilon_{\text {ax }}\right]+\left(\alpha_{s}+\zeta_{s}\right) \Delta T,
$$

where the first term refers to changes in the grating spacing caused by axial strain, the second term in brackets refers to photoelastically induced changes in the refractive index $n_{\text {core }}$ of the fiber core $(\sim 1.46$ for germanosilicate fibers), and the third and fourth terms refer to thermal expansion $\left(\sim 5 \times 10^{-7} \mathrm{~K}^{-1}\right)$ and thermo-optic $\left(\sim 7 \times 10^{-6} \mathrm{~K}^{-1}\right)$ coefficients of the fiber material, respectively (i.e., change of shape and refractive index with temperature). $P_{11} \sim 0.113$ and $P_{12} \sim 0.252$ are the photoelastic constants of silica, ${ }^{35}$ and $\varepsilon_{\text {ax }}$ and $\varepsilon_{\mathrm{r}}$ are the axial and radial strains, respectively.

When the Bragg grating is axially strained, the radial strain can be determined by Poisson's law,

$$
\varepsilon_{r}=-v_{s} \varepsilon_{\mathrm{ax}}
$$

where $v_{s}$ is the Poisson ratio of the fiber material $\left(v_{s}\right.$ $\sim 0.17$ for silica). Then the strain-temperature-towavelength relation reduces to

$$
\frac{\Delta \lambda_{B}}{\lambda_{B}}=\left(1-p_{e}\right) \varepsilon_{\mathrm{ax}}+\left(\alpha_{s}+\zeta_{s}\right) \Delta T,
$$

where $p_{e}=\left(n_{\text {core }}^{2} / 2\right)\left[P_{12}-v_{s}\left(P_{11}+P_{12}\right)\right] \approx 0.22$ for a germanosilicate fiber. At room temperature the experimental relative change in the Bragg center wavelength is then

$$
\frac{\Delta \lambda_{g}}{\lambda_{g}}=0.78 \varepsilon_{\mathrm{ax}}+7.5 \times 10^{-6} \Delta T(\mathrm{~K}) .
$$

At the wavelength of $1.3 \mu \mathrm{m}$ the temperature-towavelength coefficient is approximately $0.1 \mathrm{~K} / \mathrm{pm}$ at room temperature, and the strain-to-wavelength coefficient is approximately $1 \mu \varepsilon / \mathrm{pm} \quad(1 \mu \varepsilon=1$ $\mu$ strain $\left.=10^{-6}\right)$. Therefore a change in temperature of $0.1 \mathrm{~K}$ induces the same wavelength shift as that induced by $1 \mu$ strain. It should not be overlooked that both strain- and temperature-to-wavelength coefficients depend on temperature. For example, Meltz et al..$^{5}$ reported slopes of relative Bragg grating wavelength shift versus temperature that increased from $6.7 \times 10^{-6} \mathrm{~K}^{-1}$ (from room temperature to $150{ }^{\circ} \mathrm{C}$ ) to $9.57 \times 10^{-6} \mathrm{~K}^{-1}$ (at $450^{\circ} \mathrm{C}$ ). ${ }^{36}$ Therefore recalibrations are needed every time there is a change in the temperature range of use. (This is particularly true for all intrinsic temperaturecompensation methods previously described.)

Once the FBG is bonded onto or embedded into the test structure, the thermal expansion of the structure material causes a change in the grating period. Let $\alpha_{\text {structure }}$ be the thermal-expansion coefficient of the test material. Then Eq. (5) becomes

$$
\begin{aligned}
\frac{\Delta \lambda_{B}}{\lambda_{B}}= & \left(1-p_{e}\right) \varepsilon_{\mathrm{ax}}+\left[\zeta_{s}+\alpha_{s}+\left(1-p_{e}\right)\right. \\
& \left.\times\left(\alpha_{\text {structure }}-\alpha_{s}\right)\right] \Delta T .
\end{aligned}
$$

For $\alpha_{\text {struc }} \gg \alpha_{s}$ (for example, $\alpha_{\text {structure }}=23 \times 10^{-6}$ $\mathrm{K}^{-1}$ for aluminum), it reduces to

$$
\frac{\Delta \lambda_{B}}{\lambda_{B}}=\left(1-p_{e}\right) \varepsilon_{\mathrm{ax}}+\left[\zeta_{s}+\left(1-p_{e}\right) \alpha_{\text {structure }}\right] \Delta T .
$$

The experimental relative change in the center wavelength of a FBG bonded on aluminum is then

$$
\frac{\Delta \lambda_{g}}{\lambda_{g}}=0.78 \varepsilon_{\mathrm{ax}}+25 \times 10^{-6} \Delta T(\mathrm{~K}) .
$$

\section{Two-dimensional Stress and Strain Analysis}

In practical strain gauging the two principal strains are unknown, and the goal is to find their magnitudes and directions from a number of strain measurements taken at various angles. Therefore three independent strain measurements in three different directions along the plane (arranged at convenient angles in relation to each other) are necessary and sufficient. A fourth gauge may be added for temperature compensation.

In the following we assume that the test structure is isotropic, homogeneous, and elastic. In practice, the test structure was weakly loaded so that Hooke's law held (strain did not exceed $0.1 \%$ ). Let $x$ and $y$ be the coordinates of the plane structure and $z$ be the outward normal to the surface. Whatever the state of strain, a reference frame $(X, Y, Z)$ always exists in which shear stresses vanish and only normal stresses remain (i.e., principal stresses $\sigma_{X}$ and $\sigma_{Y}$ ). ${ }^{37}$ Now let us assume that the test structure behaves under a state of plane stress (i.e., $\sigma_{z}=\sigma_{Z}=0$ ) and that principal axes are well known. Let $\tau_{x y}$ and $\sigma_{y}$ be, respectively, the shear and normal stresses acting on a plane of outward normal $y$, and let $\sigma_{x}$ be the normal stress acting on a plane of outward normal $x$ [Fig. 1(a)]. The plane surface makes an angle $\alpha$ with respect to the principal axis $X . \quad \sigma_{X}$ and $\sigma_{Y}$ give rise to, respectively, two orthogonal stresses $C_{X}=\sigma_{X} \sin \alpha$ and $C_{Y}=\sigma_{Y} \cos \alpha$ acting on the tilted plane. A gauge aligned along the $y$ axis measures

$$
\begin{aligned}
\sigma_{y} & =C_{X} \sin \alpha+C_{Y} \cos \alpha=d^{\prime}+r^{\prime} \cos (2 \alpha), \\
\tau_{x y} & =r^{\prime} \sin (2 \alpha),
\end{aligned}
$$




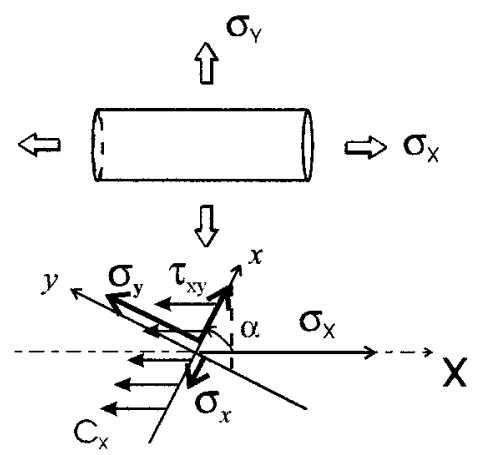

(a)

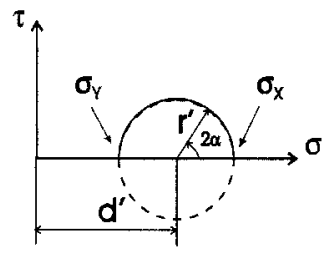

(b)
Fig. 1. Normal and shear stresses acting on a surface plane of the (a) outward normal $y$ and (b) corresponding Mohr stress circle.

where the parameters $d^{\prime}$ and $r^{\prime}$ are called the mean stress and the deviatoric stress (or stress deviator), respectively, and are expressed as follows:

$$
\begin{aligned}
d^{\prime} & =\left(\sigma_{X}+\sigma_{Y}\right) / 2, \\
r^{\prime} & =\left(\sigma_{Y}-\sigma_{X}\right) / 2 .
\end{aligned}
$$

Local strains are deduced from local stresses in Eq. (9) with Hooke's law:

$$
\varepsilon_{y}=\sigma_{y} / E=d+r \cos (2 \alpha),
$$

where $E$ is Young's modulus of the test material and $d$ and $r$ are the mean and deviatoric strains, respectively, given by

$$
\begin{gathered}
d=\left(\varepsilon_{X}+\varepsilon_{Y}\right) / 2, \\
r=\left(\varepsilon_{Y}-\varepsilon_{X}\right) / 2 .
\end{gathered}
$$

The normal and shear stresses are the two coordinates of a state of stress that can be drawn on a circle called the Mohr stress circle ${ }^{37}$ [Fig. 1(b)], described by the following equation:

$$
\left(\sigma_{y}-d^{\prime}\right)^{2}+\tau_{x y}^{2}=r^{\prime 2} .
$$

If the test structure no longer behaves under a state of plane stress, the three normal stresses are drawn on three circles called the Mohr stress tricircle. In the case of a state of plane stress, the principal strains $\varepsilon_{X}$ and $\varepsilon_{Y}$ are then

$$
\begin{aligned}
& \varepsilon_{X}=(1 / E)\left(\sigma_{X}-\nu \sigma_{Y}\right), \\
& \varepsilon_{Y}=(1 / E)\left(\sigma_{Y}-\nu \sigma_{X}\right) .
\end{aligned}
$$

The principal stresses $\sigma_{X}$ and $\sigma_{Y}$ can be derived from the principal strains

$$
\begin{aligned}
& \sigma_{X}=\left[E /\left(1-v^{2}\right)\right]\left(\varepsilon_{X}+\nu \varepsilon_{Y}\right), \\
& \sigma_{Y}=\left[E /\left(1-v^{2}\right)\right]\left(\varepsilon_{Y}+\nu \varepsilon_{X}\right),
\end{aligned}
$$

where $v$ is the Poisson ratio of the test material.

The local strain equation (12) can then be rewritten for each gauge of the rosette:

$$
\begin{aligned}
& \varepsilon_{1}=d+r \cos [2 \alpha] \quad \text { (gauge 1), } \\
& \left.\varepsilon_{2}=d+r \cos [2(\alpha+\beta)] \quad \text { (gauge } 2\right), \\
& \left.\varepsilon_{3}=d+r \cos [2(\alpha+2 \beta)] \quad \text { (gauge } 3\right),
\end{aligned}
$$

where $\alpha$ is the angular orientation of the principal axis $Y$ with respect to FBG 1. Analytical solutions for Eqs. (17) can be obtained for two types of rosettes: rectangular $\left(\beta=45^{\circ}\right)$ or delta $\left(\beta=120^{\circ}\right)$. Knowing $\beta$ and measuring $\varepsilon_{1}, \varepsilon_{2}$, and $\varepsilon_{3}$, one can determine the strain parameters $d, r$, and $\alpha$ (see Table 1). Rosettes of arbitrary angles (different from $45^{\circ}$ or $120^{\circ}$ ) are conceivable, but their practical use requires numerical resolution of Eq. (17).

An analytical form for the strain deviator can be

\begin{tabular}{|c|c|c|}
\hline $\begin{array}{l}\text { Three-Gauge } \\
\text { Rosette Types }\end{array}$ & Delta Rosette $\left(\beta=120^{\circ}\right)$ & Rectangular Rosette $\left(\beta=45^{\circ}\right)$ \\
\hline \multirow{2}{*}{ Mean strain $(d)$} & \multirow{2}{*}{$\frac{\varepsilon_{1}+\varepsilon_{2}+\varepsilon_{3}}{3}$} & $\underline{\varepsilon_{1}+\varepsilon_{3}}$ \\
\hline & & \multirow[b]{2}{*}{$\varepsilon_{1}-\varepsilon_{3}$} \\
\hline \multirow[t]{3}{*}{ Deviatoric strain $(r)$} & $\varepsilon_{2}+\varepsilon_{3}-2 \varepsilon_{1}$ & \\
\hline & $3 \cos (2 \alpha)$ & $2 \cos (2 \alpha)$ \\
\hline & $\frac{1}{3}\left(\varepsilon_{1}-\varepsilon_{3}\right)\left\{3+\left[2 \frac{\left(\varepsilon_{2}-\varepsilon_{3}\right)}{\left(\varepsilon_{1}-\varepsilon_{3}\right)}-1\right]^{2}\right\}^{1 / 2}$ & $\frac{1}{2}\left(\varepsilon_{1}-\varepsilon_{3}\right)\left\{1+\left[2 \frac{\left(\varepsilon_{2}-\varepsilon_{3}\right)}{\left(\varepsilon_{1}-\varepsilon_{3}\right)}-1\right]^{2}\right\}^{1 / 2}$ \\
\hline $\begin{array}{l}\text { Principal axis ori- } \\
\quad \text { entation }[\operatorname{tg}(2 \alpha)]\end{array}$ & $\sqrt{3} \frac{\varepsilon_{2}-\varepsilon_{3}}{2 \varepsilon_{1}-\varepsilon_{2}-\varepsilon_{3}}$ & $\overline{\varepsilon_{1}-\varepsilon_{3}}$ \\
\hline
\end{tabular}
given for both rosette types. The deviatoric formula for the delta rosette is given by

$$
r_{120^{\circ}}=\frac{1}{3}\left(\varepsilon_{1}-\varepsilon_{3}\right)\left\{3+\left[2 \frac{\left(\varepsilon_{2}-\varepsilon_{3}\right)}{\left(\varepsilon_{1}-\varepsilon_{3}\right)}-1\right]^{2}\right\}^{1 / 2},
$$

whereas the deviatoric formula for the rectangular rosette is given by

$$
r_{45^{\circ}}=\frac{1}{2}\left(\varepsilon_{1}-\varepsilon_{3}\right)\left\{1+\left[2 \frac{\left(\varepsilon_{2}-\varepsilon_{3}\right)}{\left(\varepsilon_{1}-\varepsilon_{3}\right)}-1\right]^{2}\right\}^{1 / 2} .
$$

The principal strains and stresses, as well as the angular orientation $\alpha$, can thus be computed from the measured strains $\varepsilon_{1}, \varepsilon_{2}$, and $\varepsilon_{3}$ by use of Eq. (18) or

Table 1. Parameters for Rosette Evaluation of Principal Strains $\varepsilon_{X}, \varepsilon_{Y}$, and Orientation $\alpha$ 
Eq. (19), Table 1, and the following derived from Eqs. (13a) and (13b):

$$
\begin{aligned}
& \varepsilon_{X}=d-r, \\
& \varepsilon_{Y}=d+r .
\end{aligned}
$$

\section{Bragg Grating Rosette as a}

\section{Self-Temperature-Compensated Uniaxial Strain Gauge}

Under hydrostatic pressure or temperature changes, the Mohr stress circle remains unchanged (same radius and angular orientation $2 \alpha$ ) but shifts along the $\sigma_{X}$ axis. Let us assume that the gradient in temperature is negligible and that all gauges are at the same temperature. Therefore temperature induces the same thermal-apparent strain in any direction of space, and so does hydrostatic pressure. As can be seen in Table 1, only the $d$ parameter (mean strain) depends on temperature and hydrostatic pressure, whereas $r$ (strain deviator) and $\alpha$ (angular orientation) do not because they both involve differences between local strains. As a consequence, temperature and hydrostatic effects rigorously cancel out. In the case of a uniaxial strain applied to the test structure along the $X$ direction (i.e., $\sigma_{Y}=0 ; \sigma_{X}=$ $E \varepsilon_{X}$ ), Eqs. (16) reduce merely to Hooke's and Poisson's laws, and the strain deviator can be written as

$$
|r|=\frac{\left|\varepsilon_{Y}-\varepsilon_{X}\right|}{2}=\frac{(1+\nu)}{2}\left|\varepsilon_{X}\right| .
$$

Therefore the uniaxial strain can be written as

$$
\left|\varepsilon_{X}\right|=\left(\frac{2}{1+v}\right)|r|,
$$

where $r$ is given by Eq. (18) or Eq. (19) for the delta or rectangular rosette, respectively. In the particular case of the delta rosette, the principal strain is then

$$
\varepsilon_{X}=\frac{2}{1+\nu} \frac{1}{3}\left(\varepsilon_{1}-\varepsilon_{3}\right)\left\{3+\left[2 \frac{\left(\varepsilon_{2}-\varepsilon_{3}\right)}{\left(\varepsilon_{1}-\varepsilon_{3}\right)}-1\right]^{2}\right\}^{1 / 2} \text {. }
$$

As can be seen, the rosette evaluation of the principal strain $\varepsilon_{X}$ is temperature independent.

Note that two-gauge rosettes can also be used whenever the principal axis orientation $\alpha$ is known. In the case of a $90^{\circ}$ tee rosette, the rosette strain relations are then

$\varepsilon_{1}=d+r \cos [2 \alpha]$

$\varepsilon_{2}=d+r \cos [2(\alpha+\pi / 2)]=d-r \cos [2 \alpha]$

(gauge 1),

Therefore the deviatoric strain as measured by a twogauge tee rosette type is

$$
r=\frac{\varepsilon_{1}-\varepsilon_{2}}{2 \cos [2 \alpha]} .
$$

The principal strain $\varepsilon_{X}$ can then be deduced with Eq. (22), and one can see that it is absolutely temperature independent as well.
The accuracy of the measurement of the state of strain is partly limited by spectral resolution. In fact, since three noncollinear gratings are required for one to perform the measurement, one can easily find [by differentiating Eq. (23)] that the error depends on $\alpha$ and is approximately twice to three times that of a single grating (owing to the vectorial nature of the strain information). Therefore a maximum error of approximately $3 \mu \varepsilon / \mathrm{pm}$ is anticipated for the rosette-based uniaxial strain sensor. However, a great inaccuracy arises from angular dispersion in the positioning of gauges with respect to one another. Let us suppose that one gauge is badly positioned with respect to the two other gauges. There is a small change in local strain $\Delta \varepsilon$ in comparison with that measured by the perfectly aligned gauge that can be estimated by a first-order Taylor series approximation of Eqs. (17) as a function of a small departure $\Delta \beta$ from the exact angle. Then this new strain $\varepsilon+\Delta \varepsilon$ is inserted into Eq. (18), and the corresponding change in the deviator value is estimated by a first-order Taylor-series approximation as a function of $\Delta \varepsilon$. After simplification the relative error in deviator estimation with respect to the departure angle $\Delta \beta$ is then found to be

$$
\frac{\Delta r}{r}=\frac{8}{9} \sin ^{2} \beta \sin [4(\alpha+\beta)] \Delta \beta
$$

and can be found to be, at worst $\pm 0.12 \%$ for a $0.1^{\circ}$ angular misalignment. In actual fact, two gauges can be badly positioned with respect to the third one. Therefore the relative error of the rosette sensor is actually, at worst, $\pm 0.24 \%$ for a $0.1^{\circ}$ angular misalignment.

Once $\varepsilon_{X}$ and $\alpha$ are known (Table 1), pure local strains $\varepsilon_{1}{ }^{\circ}, \varepsilon_{2}^{\circ}$, and $\varepsilon_{3}{ }^{\circ}$ can be calculated by use of the following deduced from Eqs. (17):

$$
\begin{aligned}
\frac{\varepsilon_{1}^{\circ}}{\varepsilon_{X}}= & \sin ^{2}(\alpha)-v \cos ^{2}(\alpha)=\left(\frac{1-v}{2}\right)-\left(\frac{1+v}{2}\right) \cos (2 \alpha), \\
\frac{\varepsilon_{2}^{\circ}}{\varepsilon_{X}}= & \sin ^{2}(\alpha+\beta)-v \cos ^{2}(\alpha+\beta)=\left(\frac{1-v}{2}\right)-\left(\frac{1+v}{2}\right) \\
& \times \cos (2 \alpha+2 \beta), \\
\frac{\varepsilon_{3}^{\circ}}{\varepsilon_{X}}= & \sin ^{2}(\alpha+2 \beta)-v \cos ^{2}(\alpha+2 \beta)=\left(\frac{1-v}{2}\right)-\left(\frac{1+v}{2}\right) \\
& \times \cos (2 \alpha+4 \beta) .
\end{aligned}
$$

The temperature information $T-T_{0}$ can be calculated with Eq. (7) for any grating:

$$
T-T_{0}=\left[\frac{\Delta \lambda_{i}}{\lambda_{i}}-\left(1-p_{e}\right) \varepsilon_{i}^{\circ}\right] \frac{1}{\left[\zeta_{s}+\left(1-p_{e}\right) \alpha_{\text {structure }}\right]} .
$$

The absolute temperature $T$ can then be estimated, provided that an accurate calibration of the reference temperature $T_{0}$ has been made beforehand. Since the strain $\varepsilon_{i}{ }^{\circ}$ is a function of $\varepsilon_{X}$ and $\alpha$, the relative 


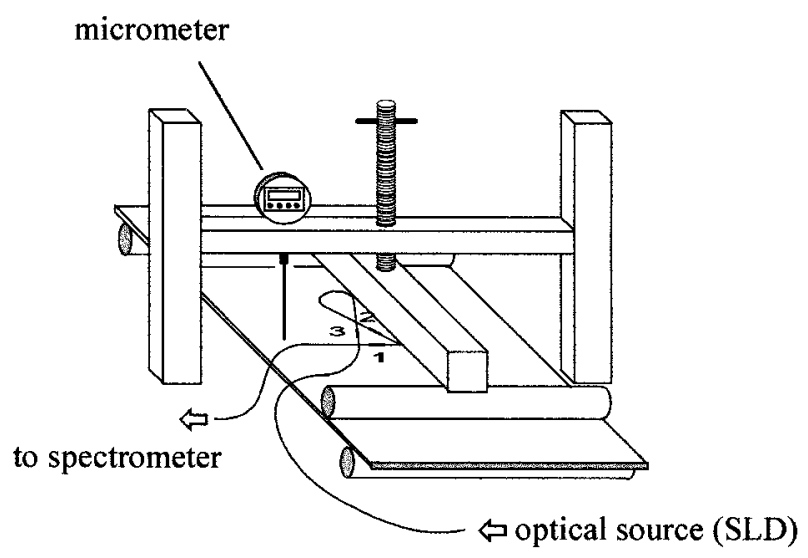

Fig. 2. Four-point bending setup: SLD, superluminescent diode.

error in temperature with respect to spectral resolution can be estimated. The calculation is lengthy but does not create any difficulty. It yields

$$
\begin{aligned}
\frac{\Delta T}{\Delta \lambda}(\mathrm{K} / \mathrm{pm})= & {\left[\frac{1}{\lambda}+\left(1-p_{e}\right)\left(\frac{1-\nu}{2}\right)\left|\frac{\delta \varepsilon_{X}}{\delta \lambda}\right|+\frac{4}{3} \frac{1}{\lambda}\right] } \\
& \times \frac{1}{\left[\zeta_{s}+\left(1-p_{e}\right) \alpha_{\text {structure }}\right]} .
\end{aligned}
$$

The third term refers to the error in angular orientation and is maximum for $\alpha=45^{\circ}$.

\section{Strain-to-Deflection Governing Equations for Plates under Bending}

A four-point bending setup was chosen for a proof-ofprinciple experiment. It is depicted in Fig. 2. It consists of two support bars fixed on an optical table, two loading bars applying a force set by a screw, and a micrometer to measure the center deflection of the test structure. Four-point bending induces a state of plane strain. Such a setup enables one to perform static measurements with great stability over long periods of time to achieve a good spectral resolution. ${ }^{20}$ Moreover, the curvature radius of the test structure is constant at the center so that the bending-induced strain is constant over the grating length (i.e., there is no spectral chirp of the grating), and there is no need for accurate positioning of the test structure on the bench. Finally, the test structure can be turned at any angle between $\pm 30^{\circ}$, and the principal axis orientation is determined by the support bars.

It can be shown from the theory of elasticity ${ }^{37,38}$ that the bending profile $w(x)$ of a beam is ruled by the well-known differential equation of equilibrium:

$$
\frac{\delta^{2} w(x)}{\delta x^{2}}=\frac{M(x)}{E I}
$$

or

$$
\frac{\delta^{4} w(x)}{\delta x^{4}}=\frac{p(x)}{E I},
$$

where $M(x)$ is the bending moment applied to the beam with respect to the abscissa $x, p(x)$ is the applied force per unit area, and $I$ is the moment of inertia of the beam cross section with respect to the $x$ and $y$ axes, which is considered constant over its length. By integrating twice and solving for boundary conditions, one can easily find the deflection $W$ at the center of the beam:

$$
W=\frac{F a}{E I}\left(\frac{a^{2}}{6}-\frac{L^{2}}{8}\right),
$$

where $L$ is the distance between support bars and $a$ is the distance between a support bar and the nearest loading bar. The upper part of the structure is under compression, whereas its lower part is under traction. The local strain at the surface of the structure increases linearly with distance from the neutral axis of the beam according to

$$
\varepsilon_{X}=-\left.\frac{(h+\Phi)}{2} \frac{\delta^{2} w(x)}{\delta x^{2}}\right|_{x=L / 2},
$$

where $\Phi$ is the diameter of the polymer-coated fiber and $h$ is the thickness of the plate. Substitution of Eq. (30a) into Eq. (32) gives

$$
\varepsilon_{X}=-\frac{(h+\Phi)}{2} \frac{M(L / 2)}{E I} .
$$

Since the bending moment at the center of the beam is $M(L / 2)=F a$, substitution of Eq. (31) into Eq. (33) finally gives the following relation between center deflection and local strain:

$$
\varepsilon_{X}=-12(h+\Phi) \frac{W}{\left(4 a^{2}-3 L^{2}\right)} .
$$

The beam-bending theory has the merits of both simplicity and ease of use because most bending problems can be solved analytically. However, owing to the overall dimensions of our Bragg grating rosette with respect to our bending setup, the test structure on which it is bonded is better described by the thin plate-bending model rather than by the beambending one. The differential equation of equilibrium is then written in two-dimensional coordinates $\mathrm{as}^{38}$

$$
\begin{aligned}
\frac{\delta^{4} w(x, y)}{\delta x^{4}}+2 \frac{\delta^{4} w(x, y)}{\delta x^{2} \delta y^{2}}+\frac{\delta^{4} w(x, y)}{\delta y^{4}} \\
=\frac{12\left(1-v^{2}\right)}{E h^{3}} p(x, y)
\end{aligned}
$$

Solutions for Eq. (35) can be obtained by the inverse method that relies on assumed solutions for $w(x, y)$ that satisfy the governing equation and the boundary conditions. A powerful method is the Fourier series (Navier or Levy solutions), but manageable solutions require one to fulfill particular boundary conditions that are not generally met in practical experiments. On the other hand, finite-element methods have 
proved to be the most flexible for determining the mechanical behavior of arbitrary test structures and mapping their state of strain. We performed platebending numerical analysis using a finite-element software (résistance des matériaux). Under a displacement imposed by the loading bars (as boundary conditions), we obtained principal strains and principal axis orientation of arbitrary points on the surface as a function of plate deflection for several orientations of the plate on the test bench.

Plate-bending behavior differs considerably from that of beam bending because of edge effects in the way that a transverse stress $\sigma_{Y}$ takes place in plate bending but is not noticeable in beam bending. Moreover, the plate takes the shape of a saddle. This behavior has been reported by several authors $^{38,39}$ and can be analytically described in simple cases. As a consequence of this, the ratio $-\varepsilon_{Y} / \varepsilon_{X}$ is inevitably less than the Poisson ratio of the test material. This apparent Poisson ratio is given by

$$
\frac{\varepsilon_{Y}}{\varepsilon_{X}}=-v_{\mathrm{app}}=\frac{\sigma_{Y}-v \sigma_{X}}{\sigma_{X}-v \sigma_{Y}} .
$$

The apparent Poisson ratio was confirmed by finiteelement analysis and was found to be nearly constant with respect to load. Indeed, the theory of elasticity always predicts a linear relation between transverse and longitudinal strains (i.e., Poisson's law always holds but with an effective Poisson ratio different from that of the test material). At any rate, as a proof of principle, the experiment is as conclusive as if it were performed on a beam (i.e., small width-tolength ratio), except that further calculations were carried out on the basis of an apparent Poisson ratio.

Moreover, numerical analysis shows that the deflection of the plate at $0^{\circ}$ orientation is identical to that of its beam equivalent [i.e., Eq. (34) holds for $0^{\circ}$ plate bending], owing to the symmetry of the distributed applied load. The experimental deflection differs from that determined by the beam-bending theory at other orientations, and results obtained by numerical analysis of plate bending must be considered instead.

\section{Experiment and Discussion}

An optical Bragg grating delta rosette was fabricated and is shown in Fig. 3(a). Each side was $35 \mathrm{~mm}$ long. The three Bragg gratings arranged at $60^{\circ}$ from one another were bonded onto an aluminum plate as the test structure [Fig. 3(b)] on the reverse side (tensile face). Seen from the top, the Bragg gratings were arranged in a counterclockwise configuration. The thickness of the plate was $h=4.28$ $\mathrm{mm}$, and its Young's modulus and Poisson ratio were $E=67.5 \mathrm{GPa}$ and $v=0.34$, respectively. The optical instrumentation has already been described in a previous publication. ${ }^{20}$ Light from a broadband superluminescent diode was launched into a singlemode fiber, in which the three Bragg gratings were photowritten at $1294.8,1296.7$, and $1297.4 \mathrm{~nm}$. The outer diameter of the polymer-coated fiber was 280

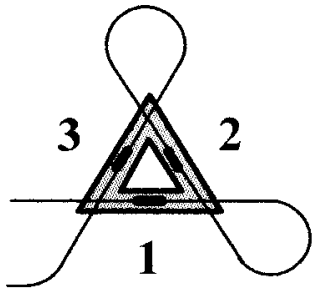

(a) fiber Bragg grating

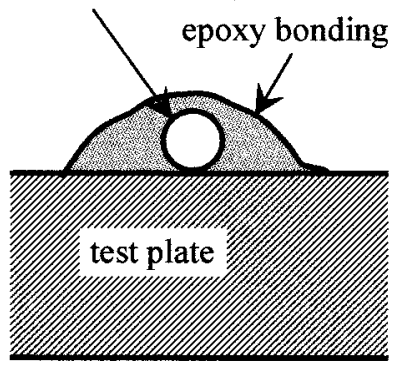

(b)
Fig. 3. Bragg grating (a) delta rosette and (b) epoxy bonding onto a metallic test plate.

$\mu \mathrm{m}$. The spectral linewidths of the Bragg grating gauges were $0.2 \mathrm{~nm}$. Bragg spectra were recorded with a 64-cm-focal-length scanning monochromator equipped with a $600-$ line $/ \mathrm{mm}$ grating. The spectral resolution was approximately $\pm 5 \mathrm{pm}$, yielding a strain resolution of $\pm 5 \mu \varepsilon$.

In a first approach we investigated the use of this rosette to determine the state of strain. Although theory [Eq. (23) and Table 1] predicts that the rosette calculation of principal strains and stresses is independent of the rosette orientation with respect to the principal axis, we intended to verify its reproducibility. With this aim in mind, we performed several measurements at various angular orientations and compared rosette evaluations of the principal strain. The plate was turned along the bench at $0^{\circ}, 14^{\circ}$, and $30^{\circ}$. For each angular orientation the plate was progressively loaded so that the deflection increased in steps of $0.5 \mathrm{~mm}$. Spectra of the three Bragg gratings were recorded for each deflection. Figures 4 and 5 show the Bragg grating wavelengths with respect to the center deflection of the plate for $0^{\circ}$ and $30^{\circ}$, respectively. Also shown are the experimental princi-

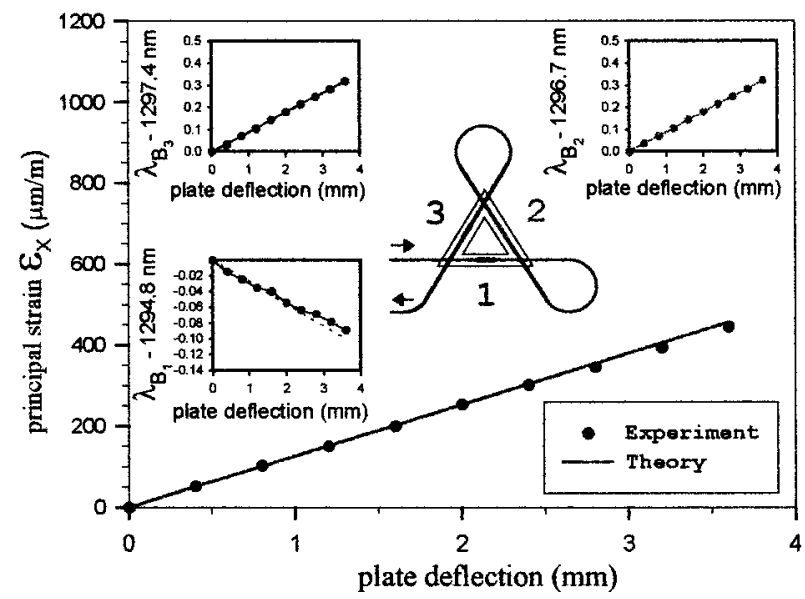

Fig. 4. Experimental and theoretical evolutions of the Bragg grating wavelengths under an increased load for $0^{\circ}$ orientation (FBG 1 is aligned along the $Y$ principal axis) and experimental and theoretical evaluations of the principal strain $\varepsilon_{X}$. 


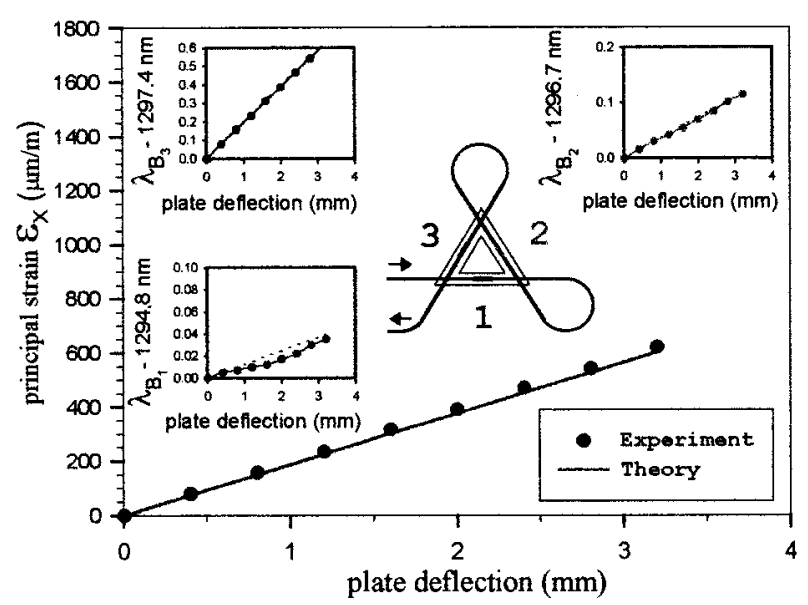

Fig. 5. Experimental and theoretical evolutions of the Bragg grating wavelengths under an increased load for $30^{\circ}$ orientation (FBG 1 is at $30^{\circ}$ with respect to the $Y$ principal axis) and experimental and theoretical evaluations of the principal strain $\varepsilon_{X}$.

pal strain $\varepsilon_{X}$ as calculated from individual Bragg grating strains with Eq. (23) and the theoretical one as obtained by numerical analysis. The theoretical local strains $\varepsilon_{1}{ }^{\circ}, \varepsilon_{2}{ }^{\circ}$, and $\varepsilon_{3}{ }^{\circ}$ were then deduced by use of Eq. (27), and the corresponding individual Bragg grating center wavelengths were obtained with Eq. (5). They are also drawn in dotted lines for comparison, and it is obvious that agreement between the experiment and the numerical analysis is good. As mentioned above, finite-element results are identical to those obtained with beam-bending calculation for $0^{\circ}$ orientation. Experimental values for $\varepsilon_{X}$ fit both the calibration (metal-foil resistance strain gauge) and the plate bending well whatever the orientation. For $0^{\circ}$ and $14^{\circ}$ orientation the dimensions of the test bench were $L=400 \mathrm{~mm}$ and $a=111 \mathrm{~mm}$. At $30^{\circ}$ orientation the dimensions were $L=295 \mathrm{~mm}$ and $a=$ $66 \mathrm{~mm}$. We estimated strain-to-deflection coefficients using Eq. (34) and finite-element modeling as well. For a given displacement imposed by loading bars, the principal strain $\varepsilon_{X}$ and deflection $W$ were reported for each angular orientation of the plate $\left(0^{\circ}\right.$, $14^{\circ}$, and $30^{\circ}$ ). Principal axis angular orientations $\alpha$ were $0^{\circ}, 11^{\circ}$, and $26.5^{\circ}$. Finite-element numerical analysis gave the principal stresses $\sigma_{X}$ and $\sigma_{Y}$, and principal strains could be deduced with Eq. (15). With Eq. (36) we obtained the apparent Poisson ratio between the two principal strains, which was 0.17. We compared the theoretical results with the Bragg grating rosette experimental evaluation of the principal strain $\varepsilon_{X}$, taking into account a strain-towavelength coefficient of $1.01 \mathrm{pm} / \mu \varepsilon$. The results are outlined in Table 2.

A quite good agreement is found between experimental evaluation of the principal strain and finiteelement numerical analysis, whereas a great difference exists when we used the beam-bending model (except for the special case of $0^{\circ}$ orientation). Figures 4 and 5 show a nonlinearity of the strain response of grating 1 (mostly submitted to the Pois-
Table 2. Principal Strain-to-Plate Deflection Coefficients $\varepsilon_{X} / W$ $(\mu \varepsilon / \mathrm{mm})$ for Various Angular Orientations of the Plate ${ }^{a}$

\begin{tabular}{cccc}
\hline $\begin{array}{c}\text { Plate } \\
\text { Orientation } \\
\text { (deg) }\end{array}$ & $\begin{array}{c}\text { Beam-Bending } \\
\text { Model }\end{array}$ & $\begin{array}{c}\text { Numerical } \\
\text { Analysis }\end{array}$ & $\begin{array}{c}\text { Experimental } \\
\text { Evaluation }\end{array}$ \\
\hline 0 & 127 & 127 & $128 \pm 2$ \\
14 & 127 & 120 & $116 \pm 2$ \\
30 & 225 & 200 & $193 \pm 2$ \\
\hline
\end{tabular}

${ }^{a}$ FBG 1, FBG 2, and FBG 3 are arranged in a counterclockwise configuration.

son strain). We believe this is due to a slight change in the Poisson ratio during plate bending (see Section 5). Since the relative change in the Poisson ratio is less than $10 \%$, it leads to a small inaccuracy in the principal strain measurement (approximately 1\%). As already described, this problem arises because of plate behavior under bending and is expected to vanish for linear structures (rods, tubular members) for which the Poisson ratio is exactly that of the structure material.

In a second approach we investigated an innovative way of using this rosette in combination with uniaxial strain to make a self-temperaturecompensated optical fiber Bragg grating-based strain gauge. Once again the plate was progressively loaded, but, as the deflection reached $1 \mathrm{~mm}$, we imposed a sudden change in temperature by heating the plate. After temperature stabilization the loading continued until the deflection reached $3.5 \mathrm{~mm}$ (Fig. 6). The corresponding strain $\varepsilon_{X}$ was approximately $435 \mu \varepsilon$. As expected, every Bragg grating center wavelength exhibited a step owing to temperature change. Heating the plate manifested itself in a thermal-apparent strain added to the real strain (shown by the dotted line) as it affected each Bragg grating wavelength. On the other hand, the experimental value of the real strain $\varepsilon_{X}$ [as calculated by Eq. (23)] remained unaffected by the change in tem-

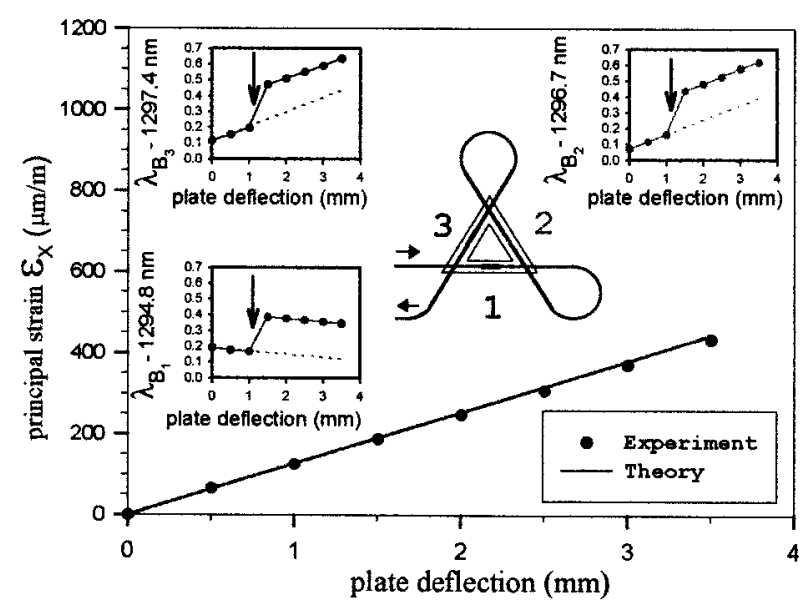

Fig. 6. Experimental and theoretical evolutions of the Bragg grating wavelengths under an increased load for $0^{\circ}$ orientation and demonstration of the self-temperature-compensated measurement of the uniaxial strain $\varepsilon_{X}$. 
perature. Since the rosette evaluation of the principal strain was independent of its angular orientation (as shown in Fig. 5), we therefore realized a uniaxial strain sensor absolutely independent of temperature as well as its positioning onto the test structure. The temperature information can be recovered as well with Eq. (28), and in the case of Fig. 6 it was found to be approximately $7 \pm 0.7^{\circ} \mathrm{C}$.

Let us assume that the Poisson ratio is accurately known. In addition to spectral resolution, a major inaccuracy stems from the fabrication tolerance of the Bragg grating rosette (by means of angular dispersion). In our experiment the rosette was accurately positioned to better than $0.1^{\circ}$ so that the relative error in uniaxial strain was expected to be less than 0.24\%, as estimated with Eq. (26). This means that, for strains ranging from several to $1000 \mu \varepsilon$, the precision in strain was limited mainly by the spectral resolution of our instrumentation $( \pm 5 \mathrm{pm})$. Therefore the error caused by spectral resolution was \pm 15 $\mu \varepsilon$, whereas the error caused by fabrication tolerance was $\pm 2.5 \mu \varepsilon$. On the other hand, for strains higher than $1000 \mu \varepsilon$, the precision in strain was determined by the angular inaccuracy (fabrication tolerance) as well. For example, for most smart-structure applications (strain range to as high as 1\%), this error would be approximately $\pm 25 \mu \varepsilon$. As a consequence of that, the real error of our rosette-based uniaxial strain sensor was estimated to be, at worst, $\pm 5.5 \mu \varepsilon$ for a strain of $1000 \mu \varepsilon$ and a given spectral resolution of $\pm 1 \mathrm{pm}$. Using Eq. (29), we found the corresponding precision in temperature to be approximately 0.14 $\mathrm{K} / \mathrm{pm}$.

\section{Conclusion}

For the first time to our knowledge, a Bragg grating rosette has been designed, fabricated, and tested. We first investigated this rosette by measuring the state of strain of a thin plate as the test structure in a four-point bending setup (i.e., measuring the principal strains and orientation of the principal axis). This rosette competed with its electrical counterparts in terms of precision and conveyed all intrinsic optical fiber sensor advantages. Satisfactory results were obtained that allowed the use of this rosette on plane structures under different loading conditions, possibly with an extra grating for temperature compensation. Other structures, such as tie rods or members of a truss (either plane or tubular), might be equipped as well.

In addition to this classical use, we have described an innovative application of this device as a selftemperature-compensated uniaxial strain sensor with no angular dependence. The uniaxial strain, the angular orientation of its principal axis, and the temperature can be accurately recovered by calculation. In support of this conception, note that most extensometric measurements involve only uniaxial strains. In-plane determination of principal axis orientation and principal strains are seldom encountered in industrial applications and are mostly restricted to engineering and laboratory experiments.
The basic idea of this self-compensation scheme is then to add more information than strictly required (i.e., three gauges instead of one) and to use the excess information to get rid of temperature, pressure, and orientation influence. This concept is, of course, of great relevance to industrial applications, since most in situ strain sensors are inherently temperature dependent and often require accurate positioning.

In contrast with other in situ temperaturecompensation methods, the FBG rosette evaluation of the uniaxial strain makes use of a rigorous calculation (i.e., deviatoric calculation) that does not rely on matrix inversion. A great advantage over alternative methods is that calculation of the uniaxial strain is inherently independent of temperature. Since it does not involve the temperature sensitivity of the transducer (e.g., FBG's), the rosette-based sensor can be used over any arbitrary temperature range without requiring frequent recalibrations because of changes in the temperature-to-wavelength coefficient of FBG's.

Finally, one can use this sensor for any isotropic test material simply by adjusting the Poisson ratio. (One must use an effective Poisson ratio instead of the structure material when making plate-bending tests.) The deviatoric equation can be calculated in real time, or the corresponding signal can be shaped by a dedicated electronic circuit. In addition to spectral resolution, a major source of inaccuracy stems from angular fabrication tolerances. The precisions of our rosette-based uniaxial strain sensor are approximately $3 \mu \varepsilon / \mathrm{pm}+2.5 \mu \varepsilon$ for a strain of $1000 \mu \varepsilon$ and $0.14 \mathrm{~K} / \mathrm{pm}$, and maximum errors of approximately $3 \mu \varepsilon / \mathrm{pm}$ and $0.11 \mathrm{~K} / \mathrm{pm}$ are anticipated for a perfectly arranged rosette-based uniaxial strain sensor. Typical strain- and temperature-to-spectralresolution relative errors are summarized in Table 3 (only for methods involving spectral measurements). Strain and temperature precisions are limited by the measurement accuracy, i.e., $1 \mu \varepsilon / \mathrm{pm}$ and $0.1 \mathrm{~K} / \mathrm{pm}$, respectively. The great limitation of most methods requiring matrix inversion is that the inversion is often poorly conditioned because of a small determinant and, as a consequence, gives large errors and low accuracy.

The satisfactory performances of this compensating scheme allow us to be confident about its many technical applications. Clear advantages over alternative methods are angular independence, high accuracy, and self-temperature compensation over a broad temperature range without recalibration. Although it has been demonstrated with FBG's as strain gauges, other transducers (e.g., interferometric) can be implemented in a similar manner.

However, as a proof of principle, this rosette has been realized in fiber form. This implementation turned out to be difficult to handle, and the angular positioning required a meticulous design. As a consequence, a fiber-based device would preferably be realized with printed circuit processes on a flexible substrate (e.g., bonded on an epoxy film). Such a 
Table 3. Comparison of Recovery Analysis Performances of Several Temperature-Compensation Methods of FBG's Involving Spectral

Measurements in Terms of Relative Error to Spectral Resolution

\begin{tabular}{|c|c|c|c|c|}
\hline \multirow[b]{2}{*}{ Compensating Method } & \multicolumn{2}{|c|}{ Relative Errors } & \multirow{2}{*}{$\begin{array}{l}\text { Extrinsic Versus } \\
\text { Intrinsic }\end{array}$} & \multirow[b]{2}{*}{ Reference } \\
\hline & Strain & Temperature & & \\
\hline $\begin{array}{l}\text { Dual overlaid FBG's at different center } \\
\text { wavelengths }\end{array}$ & $17 \mu \varepsilon / \mathrm{pm}$ & $1.7 \mathrm{~K} / \mathrm{pm}$ & Intrinsic & 22,23 \\
\hline Bragg grating in a highly birefringent fiber & Not indicated & Not indicated & Intrinsic & 24 \\
\hline Two FBG's of different cladding diameters & $17 \mu \varepsilon / \mathrm{pm}$ & $1 \mathrm{~K} / \mathrm{pm}$ & Intrinsic & 25 \\
\hline Two diffraction orders of a FBG & $17 \mu \varepsilon / \mathrm{pm}$ & $1.7 \mathrm{~K} / \mathrm{pm}$ & Intrinsic & 26 \\
\hline One rocking filter and one FBG & $40 \mu \varepsilon / 0.1 \mathrm{~nm}$ & $\begin{array}{c}0.25 \mathrm{~K} / 0.1 \\
\mathrm{~nm}\end{array}$ & Intrinsic & 27,28 \\
\hline Two FBG's and a long-period grating & Not indicated & Not indicated & Intrinsic & 29 \\
\hline Fabry-Perot interferometer and FBG & $1.25 \mu \varepsilon / \mathrm{pm}$ & $0.35 \mathrm{~K} / \mathrm{pm}$ & Extrinsic & 30 \\
\hline FBG rosette & $\begin{array}{c}3 \mu \varepsilon / \mathrm{pm}+ \\
2.5 \mu \varepsilon\end{array}$ & $0.14 \mathrm{~K} / \mathrm{pm}$ & Intrinsic & $\begin{array}{l}\text { This } \\
\text { study }\end{array}$ \\
\hline $\begin{array}{l}\text { Two FBG's mounted on opposite surfaces of } \\
\text { a cantilever }\end{array}$ & $\sim 1 \mu \varepsilon / \mathrm{pm}$ & Not measured & Extrinsic & 32 \\
\hline Passive temperature-compensating package & $\begin{array}{c}70 \mu \varepsilon \text { error } \\
\text { on } 120^{\circ} \mathrm{C} \\
\text { range }\end{array}$ & Not measured & Extrinsic & 33,34 \\
\hline
\end{tabular}

substrate of small thickness is required to allow for large elongations (several 10,000 $\mu \varepsilon$ ) without significant alteration of the strain distribution in the structure under test. An even more efficient technology could be integrated optics on polymers as a means of achieving high accuracy in fabrication tolerances, reproducibility, and integration in a mass-produced process. Indeed, polymer substrates have been shown to be photosensitive, and channel waveguides and gratings have been photowritten in such substrates. ${ }^{11}$ Fibers or channel waveguides of great index difference between the core and the cladding $\left(10^{-2}\right)$ do not suffer significant curvature losses for a bending radius as low as several millimeters. Consequently, we can anticipate that the design and elaboration of integrated rosettes of overall dimensions of approximately $10 \times 10 \mathrm{~mm}^{2}$ or less may be possible. This compactness is comparable with or even smaller than that of their resistive foil counterparts.

As a strain transducer the Bragg grating can be used to sense many other physical parameters by means of an appropriate strain-transducing mechanism. For example, one might use magnetostrictive substrates to measure the amplitude of a magneticfield vector ${ }^{40}$ or, alternatively, an electric current (for example, in a transformer). Such a Bragg grating rosette-based sensor would be self-temperature compensated and independent of its angular orientation with respect to the field vector. One might use piezoelectric substrates or coatings as well to measure an electric-field vector. A section of fiber containing a Bragg grating can be bonded onto or embedded into materials that change size or shape in the presence of the electric field. One could also use FBG's coated with a piezoelectric polymer jacket [e.g., poly(vinylidene fluoride)] to perform such a task.

We thank C. Prioul and M. Pasquet of Ecole Centrale des Arts et Manufactures, Chatenay-Malabry,
France, for technical support with the finite-element numerical analysis résistance des matériaux software obtained from Université du Mans, France) and W. W. Morey of 3M Bragg grating technology (specialty optical fibers) for fruitful collaborations and for providing us with Bragg gratings.

\section{References}

1. I. Bennion, J. A. R. Williams, L. Zhang, K. Sugden, and N. J. Doran, "U.V.-written in-fibre Bragg gratings," Opt. Quantum Electron. 28, 93-135 (1996).

2. R. J. Campbell and R. Kashyap, "The properties and applications of photosensitive germanosilicate fibers," Int. J. Optoelectron. 9, 33-57 (1994).

3. W. W. Morey, G. Meltz, and W. H. Glenn, "Bragg grating temperature and strain sensors," in Sixth Optical Fiber Sensor Conference, Paris, France, Springer Proc. 44, 526-531 (1989).

4. K. O. Hill, Y. Fujii, D. C. Johnson, and B. S. Kawasaki, "Photosensitivity in optical fiber waveguides: application to reflection filter fabrication," Appl. Phys. Lett. 32, 647-649 (1978).

5. G. Meltz, W. W. Morey, and W. H. Glenn, "Formation of Bragg gratings in optical fibers by a transverse holographic method," Opt. Lett. 14, 823-825 (1989).

6. K. O. Hill, B. Malo, F. Bilodeau, D. C. Johnson, and J. Albert, "Bragg gratings fabricated in monomode photosensitive optical fiber by UV exposure through a phase mask," Appl. Phys. Lett. 62, 1035-1037 (1993).

7. P. J. Lemaire, R. M. Atkins, V. Mizrahi, and W. A. Reed, "High pressure $\mathrm{H}_{2}$-loading as a technique for achieving ultrahigh U.V. photosensitivity in $\mathrm{GeO}_{2}$-doped optical fibres," Electron. Lett. 29, 1191-1193 (1993).

8. M. M. Broer, R. L. Cone, and J. R. Simpson, "Ultravioletinduced distributed-feedback gratings in $\mathrm{Ce}^{3+}$-doped silica optical fibers," Opt. Lett. 16, 1391-1393 (1991).

9. L. Dong, J. L. Cruz, J. A. Tucknott, L. Reekie, and D. N. Payne, "Strong photosensitive gratings in tin-doped phosphosilicate optical fibers," Opt. Lett. 20, 1982-1984 (1995).

10. H. Poignant, S. Boj, E. Delevaque, M. Monerie, T. Taunay, P. Niay, P. Bernage, and W. X. Xie, "Efficiency and thermal behavior of cerium-doped fluorozirconate glass fibre Bragg gratings," Electron. Lett. 30, 1339-1341 (1994).

11. B. M. Monroe and W. K. Smothers, "Photopolymers for holog- 
raphy and waveguide applications," in Polymers for Lightwave and Integrated Optics: Technology and Applications (Marcel Dekker, New York, 1992), Chap. 5; B. L. Booth, "Optical interconnection polymers," in Polymers for Lightwave and Integrated Optics: Technology and Applications (Marcel Dekker, New York, 1992), Chap. 9

12. R. M. Measures, "Fiber optic sensing for smart materials and structures," in Eighth Optical Fiber Sensor Conference, F. Leonberger and A. Dandridge, eds. (IEEE, New York, 1992), pp. 366-367.

13. P. D. Foote, "Fibre Bragg grating strain sensors for aerospace smart structures," in Second European Conference on Smart Structures and Materials, A. McDonald, P. T. Gardiner, B. Culshaw, and R. S. McEwen, eds., Proc. SPIE 2361, 290-293 (1994).

14. W. W. Morey, G. A. Ball, and H. Singh, "Applications of fiber grating sensors," in Fiber Optics and Laser Sensors XIV, R. P. De Paula and J. W. Berthold III, eds., Proc. SPIE 2839, 2-7 (1996).

15. C. D. Butter and G. B. Hocker, "Fiber optics strain gauge," Appl. Opt. 17, 2867-2869 (1978).

16. J. S. Sirkis and C. E. Taylor, "Interferometric-fibre-optic strain sensor," Exp. Mech. 28, 170-176 (1988).

17. J. S. Sirkis and H. W. Haslach, "Interferometric strain measurement by arbitrarily configured, surface-mounted optical fibers," J. Lightwave Technol. 8, 1497-1503 (1990).

18. T. Valis, D. Hogg, and R. M. Measures, "Fiber optic FabryPerot strain rosettes," Smart Mater. Struct. 1, 227-232 (1992).

19. S. W. Case, J. J. Lesko, B. R. Fogg, and J. P. Carman, "Embedded extrinsic Fabry-Perot fiber optic strain rosette sensors," J. Intelligent Mater. Syst. Struct. 5, 412-417 (1994).

20. P. Ferdinand, O. Ferragu, J. L. Lechien, B. Lescop, S. Magne, V. Marty, S. Rougeault, G. Kotrosios, V. Neuman, Y. Depeursinge, J. B. Michel, M. Van Uffelen, D. Varelas, H. Berthou, G. Pierre, Ch. Renouf, B. Jarret, Y. Verbandt, W. Stevens, M. R. H. Vœet, and D. Toscano, "Mine operating accurate stability control with optical fiber sensing and Bragg grating technology: the BRITE-EuRam STABILOS project," J. Lightwave Technol. 13, 1303-1313 (1995).

21. M. G. Xu, L. Dong, L. Reekie, J. A. Tucknott, and J. L. Cruz, "Temperature-independent strain sensor using a chirped Bragg grating in a tapered optical fibre," Electron. Lett. 31, 823-825 (1995).

22. M. G. Xu, J. L. Archambault, L. Reekie, and J. P. Dakin, "Discrimination between strain and temperature effects using dual-wavelength fibre grating sensors," Electron. Lett. 30, 1085-1087 (1994).

23. E. Udd, D. Nelson, C. Lawrence, J. R. Spingarn, and B. Ferguson, "Three axis strain and temperature sensor," in Eleventh Optical Fiber Sensor Conference, Y. Ohtsuka and T. Yoshino, eds. (Japan Society of Applied Physics, Sapporo, Japan, 1996), pp. $244-247$

24. J. R. Dunphy, G. Meltz, M. Varasi, A. Vannucci, M. Signorazzi, P. Ferraro, S. I. Imparato, and C. Voto, "Embedded optical sensor capable of strain and temperature measurement using a single diffraction grating," U.S. patent 5,399,854 (21 March 1994).

25. S. W. James, M. L. Dockney, and R. P. Tatam, "Simultaneous independent temperature and strain measurement using infibre Bragg grating sensors," Electron. Lett. 32, 1133-1134 (1996).

26. G. P. Brady, K. Kalli, D. J. Webb, D. A. Jackson, L. Zhang, and I. Bennion, "Recent developments in optical fibre sensing using fibre Bragg gratings," in Fiber Optic and Laser Sensors XIV, R. P. De Paula and J. W. Berthold III, eds., Proc. SPIE 2839, 8-19 (1996).

27. S. E. Kanellopoulos, V. A. Handerek, and A. J. Rogers, "Simultaneous strain and temperature sensing employing a photogenerated polarisation coupler and low-order modes in an elliptically cored optical fibre," Electron. Lett. 30, 1786-1787 (1994).

28. S. E. Kanellopoulos, V. A. Handerek, and A. J. Rogers, "Simultaneous strain and temperature sensing with photogenerated in-fiber gratings," Opt. Lett. 20, 333-335 (1995).

29. H. J. Patrick, G. M. Williams, A. D. Kersey, J. R. Pedrazzani, and A. M. Vengsarkar, "Hybrid fiber Bragg grating/long period fiber grating sensor for strain/temperature discrimination," IEEE Photon. Technol. Lett. 8, 96-99 (1996).

30. T. Liu, G. Fernando, Y. J. Rao, D. A. Jackson, L. Zhang, and I. Bennion, "Simultaneous strain and temperature measurements in composites using a multiplexed fibre Bragg grating sensor and an extrinsic Fabry-Perot sensor," in Smart Sensing, Processing, and Instrumentation, R. O. Claus, ed., Proc. SPIE 3042, 203-212 (1997).

31. W. Jin, W. C. Michie, G. Thursby, M. Konstantaki, and B. Culshaw, "Simultaneous strain and temperature recovery: error analysis," in Eleventh Optical Fiber Sensor Conference, Y. Ohtsuka and T. Yoshino, eds. (Japan Society of Applied Physics, Sapporo, Japan, 1996), pp. 116-119.

32. M. G. Xu, J. L. Archambault, L. Reekie, and J. P. Dakin, "Structural bending sensor using fibre gratings," in Fiber Optic and Laser Sensors XII, R. P. De Paula, ed., Proc. SPIE 2292, 407-413 (1994).

33. W. W. Morey and W. L. Glomb, "Incorporated Bragg filter temperature compensated optical waveguide device," U.S. patent 5,042,898 (27 August 1991).

34. G. W. Yoffe, P. A. Krug, F. Ouellette, and D. A. Thorncraft, "Passive temperature-compensating package for optical fiber gratings," Appl. Opt. 34, 6859-6861 (1995).

35. A. Bertholds and R. Dandliker, "Determination of the individual strain-optic coefficients in single-mode optical fibers," J. Lightwave Technol. 6, 17-20 (1988).

36. G. Meltz and W. W. Morey, "Bragg grating formation and germanosilicate fiber photosensitivity," in International Workshop on Photoinduced Self-Organization Effects in Optical Fiber, F. Ouellette, ed., Proc. SPIE 1516, 185-199 (1991).

37. S. P. Timoshenko and J. N. Goodier, Theory of Elasticity (McGraw-Hill, New York, 1970).

38. A. C. Ugural, Stresses in Plates and Shells (McGraw-Hill, New York, 1981)

39. A. S. Saada, Elasticity-Theory and Application (Pergamon, New York, 1974).

40. X. Z. Lin, Y. Zhang, H. L. An, and H. D. Liu, "Electrically tunable singlemode fibre Bragg reflective filter," Electron. Lett. 30, 887-888 (1994). 